\section{Web-based submission of manuscripts to The Journal of Headache and Pain}

The dramatic increase during the last decade in research on genetics, socio-economics, pharmacology and public healthcare issues related to headache disorders produced a boost in the submission of manuscripts to both general and specialty journals, with the unavoidable appearance of new journals, an increase in the number of issues of existing ones, and an overall delay in the processing of the submitted manuscripts. Besides this was a common situation existing in several journals on handling the peer review work flow of hard copies of numberless manuscripts, it certainly disagreed with a publishing needs of researchers based on quality, indexing, ranking and mostly swiftness.

The online possibility to submit/review/resend and accept/revise/reject decision ferried across the river the undeceived enthusiasms of the young researchers. The reduction in time from submission to final decision ranked to first level on the request of the scientific writers. The possibility to track one's own paper rendered transparency to the immense work done by editors. Thus the author may accept more easily any final decision on his or her manuscript.

This Web-based possibility of an almost immediate publication of new data may be also easily coupled with use of the print-on-demand of the full paper produced a faster increasing visibility of the customer work. The today need to capture new data to support our research outcomes, metadata, hypothesis, etc. provided an explosive success of independent platforms in facilitating and allowing for the most current science to be presented and disseminated. In addition to the smoothly benefits that customers of The Journal of Headache and Pain may reach by using this virtual service the lower cost in expenditure for editing staff, priority/courier mail for both Editorial Office and customers have to be strongly suggested.

Our Editorial Board clearly indicated this new way to managing The Journal of Headache and Pain to foster the dream of both unleashed creativity and the rigorous methodology to be represented in real time in the virtual battle of the research discovery. This new and innovative on-line procedure for paper submission will, however, operate along side the more traditional practice, i.e. submission by mail.

In a short time our journal will provide this innovative service to our readership, and to our referees, so any author/reviewer may have access to submission area to send/review manuscripts and the related ancillary files only by using his or her user ID and password.

I hope that this innovation will help in providing a broader and faster dissemination of the running data emerging from this flourishing area of clinical and experimental medicine applied to headache. I firmly look for this and this Web-enhanced facility will be soon available for our readership. 